Research Article

\title{
Double Integral-Based Method for Ranking Intuitionistic Multiplicative Sets and Its Application in Selecting Logistics Transfer Station
}

\author{
Zhiyong Tian $\mathbb{D}^{1}$ and Jian Lin $\mathbb{D}^{2,3}$ \\ ${ }^{1}$ Beijing Intelligent Logistics System Collaborative Innovation Center, Beijing Wuzi University, Beijing 101149, China \\ ${ }^{2}$ College of Computer and Information Sciences, Fujian Agriculture and Forestry University, Fuzhou 350002, China \\ ${ }^{3}$ Institute of Big Data for Agriculture and Forestry, Fujian Agriculture and Forestry University, Fuzhou 350002, China
}

Correspondence should be addressed to Jian Lin; linjian36@163.com

Received 28 October 2020; Revised 15 December 2020; Accepted 26 December 2020; Published 13 January 2021

Academic Editor: Mingwei Lin

Copyright (c) 2021 Zhiyong Tian and Jian Lin. This is an open access article distributed under the Creative Commons Attribution License, which permits unrestricted use, distribution, and reproduction in any medium, provided the original work is properly cited.

Intuitionistic multiplicative sets can be applied in many practical situations, most of which are based on ranking of intuitionistic multiplicative numbers. This study develops an integral method for ranking intuitionistic multiplicative numbers based on the new definitions of multiplicative score function and accuracy function. The ranking method considers both the risk preference and infinitely many possible values in feasible region. Some reasonable properties of multiplicative score function and accuracy function are studied, respectively. We construct a total order relation on the set of intuitionistic multiplicative numbers. The multiplicative score function and accuracy function are utilized to select the optimal logistics transfer station. A comparison example is developed to highlight the advantage of the risk preference-based ranking method.

\section{Introduction}

Intuitionistic fuzzy set [1] contains three aspects of preference information, and it can represent the characteristics of things more comprehensively in reality. Since the concept of the intuitionistic fuzzy set was put forward, the related theory and application have become the research hotspots among various fields, such as intelligent computing [2-4], cluster analysis [5-7], decision-making [8-12], green supplier selection [13-15], and so on.

Representing the symmetric and uniform preferences of decision makers only, the intuitionistic fuzzy set is often inconsistent with human intuition in actual life. To overcome this in constructing intuitionistic preference relation, Xia et al. [16] defined the intuitionistic multiplicative set based on the 1/9-9 scale. The intuitionistic multiplicative set can effectively express asymmetric and uneven preference information that appears in many practical decision-making problems. For example, the intuitionistic multiplicative set is very suitable to describe the law of diminishing marginal utility in economics [17-19]. So far, a series of important results have been achieved in the theory and application of intuitionistic multiplicative sets. Xu [20] proposed the expected intuitionistic multiplicative preference relation based on intuitionistic multiplicative numbers. The priority weight intervals were derived by the geometric aggregation operator and the error propagation formula. Xu and Xia [21] defined the intuitionistic multiplicative preference relation by considering two parts of information describing the intensity degrees. Choquet integral was used to aggregate the intuitionistic multiplicative intuitionistic multiplicative numbers. Jiang and $\mathrm{Xu}$ [22] proposed two kinds of methods to ranking alternatives; meanwhile, transformation mechanism and aggregation operators were developed. $\mathrm{Yu}$ and Fang [23] fused the intuitionistic multiplicative information by defining the concepts of two aggregation operators. Yu and $\mathrm{Xu}$ [24] extended the intuitionistic multiplicative set to the intuitionistic multiplicative triangular fuzzy set. The operational laws and desirable properties are studied. Jiang et al. [25] proposed the interval-valued intuitionistic 
multiplicative set based on an unsymmetrical scale. The comparison laws of interval-valued intuitionistic multiplicative numbers were given. Jiang et al. [19] investigated an approach for group decision-making based on incomplete intuitionistic multiplicative preference relation. Ren et al. [26] applied the intuitionistic multiplicative numbers to the analytic hierarchy process. Qian and Niu [27] defined some effective operational laws of intuitionistic multiplicative numbers. Moreover, two useful aggregation operators were proposed. Jiang et al. [28] studied some universal distances based on the classical Minkowski distance. Garg [29] defined some distance measures between two or more intuitionistic multiplicative preference relations. Zhang and Pedrycz [30] checked the consistency of intuitionistic multiplicative preference relation. The generating weights were derived based on the consistent preference relation. Zhang and Guo [31] analyzed the consistency definition of intuitionistic multiplicative preference relation and established a linear programming-based algorithm to improve the flaws. Ma and $\mathrm{Xu}$ [32] utilized the parameterized hyperbolic scale to describe the preference values. Liao et al. [33] investigated some novel distance measures between intuitionistic multiplicative sets. Jin et al. [34] derived the normalized intuitionistic multiplicative weights from consistent intuitionistic multiplicative preference relation. Zhang and Pedrycz [35] presented the concept of intuitionistic multiplicative preference to deal with multicriteria group decision-making problems. Mamata [36] estimated initial values for all missing entries based on incomplete interval-valued intuitionistic multiplicative preference relation. Zhang and Chen [37] studied decision-making with incomplete intuitionistic multiplicative preference relations. A reasonable consistency of incomplete intuitionistic multiplicative preference relation was introduced.

Many practical applications of intuitionistic multiplicative sets were based on the ranking of intuitionistic multiplicative numbers. Xia et al. [16] defined the score and accuracy functions of intuitionistic multiplicative numbers. To construct a total order, some comparison laws were introduced in detail. Jiang et al. [38] developed two approaches for ranking intuitionistic multiplicative numbers based on distance measures. In order to consider the decision maker's personal preference in the process of ranking, Chen [39] proposed a ranking formula of intuitionistic fuzzy numbers with preference parameters. However, the existing method for ranking intuitionistic multiplicative numbers do not take into account the preference information of decision makers. The above two ranking methods only consider the single point value and ignore the infinite number of possible values, which makes that the ranking result may be distorted and invalid in some cases. To overcome the shortages of existing ranking methods, this study studies a novel method for ranking intuitionistic multiplicative numbers based on the risk preference of decision makers. The proposed ranking method takes all the potential possible values in feasible region into account. Therefore, the ranking results obtained by the new method are more reliable and reasonable. Moreover, the risk preference-based ranking method is applied to select the optimal logistics transfer station with intuitionistic multiplicative information.
The rest of this study is structured as follows. Section 2 reviews some related concepts on the intuitionistic multiplicative set. In Section 3, the accuracy function and score function of intuitionistic multiplicative numbers are defined based on risk preference of the decision maker. Section 4 proposes the total order among intuitionistic multiplicative numbers. Numerical example and comparison are calculated in Section 5. Conclusion and further study are stated in Section 6.

\section{Preliminaries}

For the convenience of the follow-up discussion, intuitionistic multiplicative number and its order relation are reviewed as follows.

Definition 1 (See [16]). Let set $X$ be fixed. An intuitionistic multiplicative set $M$ over $X$ is defined as

$$
\tilde{M}=\left\{<x, \rho_{M}(x), \sigma_{M}(x)>\mid x \in X\right\},
$$

where $\rho_{M}(x)$ and $\sigma_{M}(x)$ are the membership information and nonmembership information of $x$ with respect to intuitionistic multiplicative set $M$, respectively, such that $\rho_{M}(x), \sigma_{M}(x) \in[(1 / 9), 9]$, and $0<\rho_{M}(x) \sigma_{M}(x) \leq 1$. The hesitation or uncertain information of $x$ corresponding to $M$ is denoted by $\tau_{M}(x)=\left(1 /\left(\rho_{M}(x) \sigma_{M}(x)\right)\right)$.

Clearly, $\forall x \in X$, and $1 \leq \tau_{M}(x) \leq 9^{2}$. An intuitionistic multiplicative number (IMN) can be represented by $\widetilde{\alpha}=\left(\rho_{\alpha}, \sigma_{\alpha}\right)$, such that $\rho_{\alpha}, \sigma_{\alpha} \in[(1 / 9), 9], 0<\rho_{\alpha} \sigma_{\alpha} \leq 1$, and $\tau_{\alpha}=\tau(\widetilde{\alpha})=\left(1 /\left(\rho_{\alpha} \sigma_{\alpha}\right)\right)$. IM denotes the set of all IMNs.

Definition 2 (See [16]). Let $\widetilde{\alpha}=\left(\rho_{\alpha}, \sigma_{\alpha}\right)$ be an intuitionistic multiplicative number. The score function and accuracy function of $\tilde{\alpha}$ are expressed by

$$
\begin{gathered}
S_{\mathrm{Xia}}(\widetilde{\alpha})=\frac{\rho_{\alpha}}{\sigma_{\alpha}}, \\
A_{\mathrm{Xia}}(\widetilde{\alpha})=\rho_{\alpha} \sigma_{\alpha} .
\end{gathered}
$$

Definition 3 (Reference [16]). Let $\tilde{\alpha}_{1}=\left(\rho_{1}, \sigma_{1}\right)$ and $\widetilde{\alpha}_{2}=\left(\rho_{2}, \sigma_{2}\right)$ be two intuitionistic multiplicative numbers. The order relation between $\widetilde{\alpha}_{1}$ and $\widetilde{\alpha}_{2}$ is defined as follows:

(1) If $S_{\mathrm{Xia}}\left(\widetilde{\alpha}_{1}\right)>S_{\mathrm{Xia}}\left(\widetilde{\alpha}_{2}\right)$, then $\widetilde{\alpha}_{1}>_{\mathrm{Xia}} \widetilde{\alpha}_{2}$

(2) If $S_{\mathrm{Xia}}\left(\widetilde{\alpha}_{1}\right)=S_{\mathrm{Xia}}\left(\widetilde{\alpha}_{2}\right)$, then
(a) If $A_{\mathrm{Xia}}\left(\widetilde{\alpha}_{1}\right)=A_{\mathrm{Xia}}\left(\widetilde{\alpha}_{2}\right)$, then $\widetilde{\alpha}_{1}=\widetilde{\alpha}_{2}$
(b) If $A_{\mathrm{Xia}}\left(\widetilde{\alpha}_{1}\right)>A_{\mathrm{Xia}}\left(\widetilde{\alpha}_{2}\right)$, then $\tilde{\alpha}_{1}>_{\mathrm{Xia}} \widetilde{\alpha}_{2}$

\section{The Multiplicative Accuracy and Score Functions of Intuitionistic Multiplicative Numbers}

In the following, we will define the multiplicative accuracy function and score function with considering the risk preference of the decision maker. 


\subsection{The Multiplicative Accuracy Function Based on Risk Preference}

Definition 4. Let $\tilde{\alpha}=\left(\rho_{\alpha}, \sigma_{\alpha}\right)$ be an intuitionistic multiplicative number. The feasible region after transformation of $\widetilde{\alpha}$ is denoted by

$$
R_{\alpha}=\left\{(x, y) \mid \bar{\rho}_{\alpha} \leq x \leq-\bar{\sigma}_{\alpha}, \bar{\sigma}_{\alpha} \leq y \leq-x\right\},
$$

where $\bar{\rho}_{\alpha}=\ln \rho_{\alpha}, \bar{\sigma}_{\alpha}=\ln \sigma_{\alpha}$, and $\bar{\tau}_{\alpha}=\ln \tau_{\alpha}$, respectively.

Since $\rho_{\alpha} \sigma_{\alpha} \tau_{\alpha}=1$, we have $\ln \rho_{\alpha}+\ln \sigma_{\alpha}+\ln \tau_{\alpha}=0$. It follows that $\bar{\rho}_{\alpha}+\bar{\sigma}_{\alpha}+\bar{\tau}_{\alpha}=0$. Obviously, (4) can be further written as

$$
R_{\alpha}=\left\{(x, y) \mid \ln \rho_{\alpha} \leq x \leq-\ln \sigma_{\alpha}, \ln \sigma_{\alpha} \leq y \leq-x\right\},
$$

where $R_{\alpha}$ represents the mapping feasible region with respect to intuitionistic multiplicative numbers $\widetilde{\alpha}$. The mapping feasible region $R_{\alpha}$ is shown in Figure 1.

Definition 5. Let $\tilde{\alpha}=\left(\rho_{\alpha}, \sigma_{\alpha}\right)$ be an intuitionistic multiplicative number. The multiplicative accuracy function of $\tilde{\alpha}$ based on risk preference is expressed as

$$
A_{\lambda}(\widetilde{\alpha})=e^{\left(\iint_{R_{\alpha}}(\lambda x+(1-\lambda) y) \mathrm{d} x \mathrm{~d} y /\left(M\left(R_{\alpha}\right)\right)\right)},
$$

where $R_{\alpha}$ is the mapping feasible region of intuitionistic multiplicative number $\widetilde{\alpha}$, and $M\left(R_{\alpha}\right)$ is the area of mapping

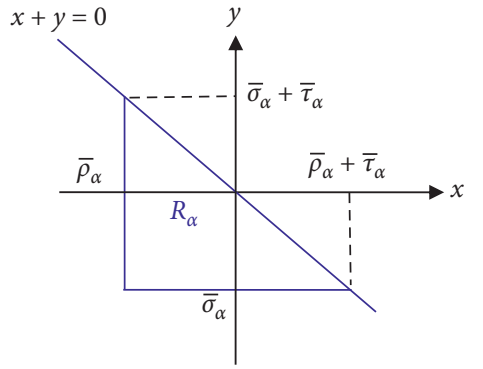

FIGURE 1: The mapping feasible region with respect to. $\widetilde{\alpha}$.

feasible region $R_{\alpha}$. The parameter $\lambda$ reflects the risk tendency of the decision maker. When $\lambda \in(0.5,1]$, the decision maker is a risk lover. When $\lambda \in[0,0.5)$, the decision maker is averse to risk. Especially, the attitude of the decision maker is neutral to the risk when $\lambda=0.5$.

$A_{\lambda}(\widetilde{\alpha})$ represents the geometric average of accuracy function over all feasible values. From (5), we have

$$
M\left(R_{\alpha}\right)=\frac{1}{2} \bar{\tau}_{\alpha}^{2}=\frac{1}{2}\left(\bar{\rho}_{\alpha}+\bar{\sigma}_{\alpha}\right)^{2}
$$

According to the operational properties of double integral, we have

$$
\begin{aligned}
\iint_{R_{\alpha}}(\lambda x+(1-\lambda) y) \mathrm{d} x \mathrm{~d} y & =\int_{\bar{\rho}_{\alpha}}^{-\bar{\sigma}_{\alpha}}\left(\int_{\bar{\sigma}_{\alpha}}^{-x}(\lambda x+(1-\lambda) y) \mathrm{d} y\right) \mathrm{d} x \\
& =\int_{\bar{\rho}_{\alpha}}^{-\bar{\sigma}_{\alpha}}\left(\frac{1-3 \lambda}{2} x^{2}-\lambda \bar{\sigma}_{\alpha} x+(\lambda-1) \frac{\left(\bar{\sigma}_{\alpha}\right)^{2}}{2}\right) \mathrm{d} x \\
& =\frac{2-3 \lambda}{6}\left(\bar{\sigma}_{\alpha}\right)^{3}-\frac{1-3 \lambda}{6}\left(\bar{\rho}_{\alpha}\right)^{3}+\frac{\lambda \bar{\sigma}_{\alpha}}{2}\left(\bar{\rho}_{\alpha}\right)^{2}-\frac{\lambda-1}{2}\left(\bar{\sigma}_{\alpha}\right)^{2} \bar{\rho}_{\alpha} .
\end{aligned}
$$

Based on (6), it follows that

$$
\begin{aligned}
A_{\lambda}(\widetilde{\alpha}) & =e^{\left(\iint_{R_{\alpha}}(\lambda x+(1-\lambda) y) \mathrm{d} x \mathrm{~d} y /\left(M\left(R_{\alpha}\right)\right)\right)}=e^{\left(((2-3 \lambda) / 6)\left(\bar{\sigma}_{\alpha}\right)^{3}-((1-3 \lambda) / 6)\left(\bar{\rho}_{\alpha}\right)^{3}+\left(\left(\lambda \bar{\sigma}_{\alpha}\right) / 2\right)\left(\bar{\rho}_{\alpha}\right)^{2}-((\lambda-1) / 2)\left(\bar{\sigma}_{\alpha}\right)^{2} \bar{\rho}_{\alpha} /(1 / 2)\left(\bar{\rho}_{\alpha}+\bar{\sigma}_{\alpha}\right)^{2}\right)} \\
& =e^{\left(\left(2\left(\bar{\sigma}_{\alpha}\right)^{3}-\left(\bar{\rho}_{\alpha}\right)^{3}+3\left(\bar{\sigma}_{\alpha}\right)^{2} \bar{\rho}_{\alpha}+3 \lambda\left(\left(\bar{\rho}_{\alpha}\right)^{3}-\left(\bar{\sigma}_{\alpha}\right)^{3}+\left(\bar{\rho}_{\alpha}\right)^{2} \bar{\sigma}_{\alpha}-\left(\bar{\sigma}_{\alpha}\right)^{2} \bar{\rho}_{\alpha}\right)\right) /\left(3\left(\bar{\rho}_{\alpha}+\bar{\sigma}_{\alpha}\right)^{2}\right)\right)} \\
& =e^{\left(\left(\left(2 \bar{\sigma}_{\alpha}-\bar{\rho}_{\alpha}\right)\left(\bar{\sigma}_{\alpha}+\bar{\rho}_{\alpha}\right)^{2}+3 \lambda\left(\bar{\rho}_{\alpha}-\bar{\sigma}_{\alpha}\right)\left(\bar{\sigma}_{\alpha}+\bar{\rho}_{\alpha}\right)^{2}\right) /\left(3\left(\bar{\rho}_{\alpha}+\bar{\sigma}_{\alpha}\right)^{2}\right)\right)} \\
& =e^{(1 / 3)\left(2 \bar{\sigma}_{\alpha}-\bar{\rho}_{\alpha}\right)+\lambda\left(\bar{\rho}_{\alpha}-\bar{\sigma}_{\alpha}\right)}=e^{\lambda\left(\left(2 \bar{\rho}_{\alpha}-\bar{\sigma}_{\alpha}\right) / 3\right)+(1-\lambda)\left(\left(2 \bar{\sigma}_{\alpha}-\bar{\rho}_{\alpha}\right) / 3\right)}
\end{aligned}
$$


Since $\bar{\rho}_{\alpha}=\ln \rho_{\alpha}$ and $\bar{\sigma}_{\alpha}=\ln \sigma_{\alpha}$, we have

$$
\begin{aligned}
A_{\lambda}(\widetilde{\alpha}) & =e^{\lambda\left(\left(2 \ln \rho_{\alpha}-\ln \sigma_{\alpha}\right) / 3\right)+(1-\lambda)\left(\left(2 \ln \sigma_{\alpha}-\ln \rho_{\alpha}\right) / 3\right)} \\
& =e^{(\lambda / 3) \ln \left(\left(\rho_{\alpha}\right)^{2} / \sigma_{\alpha}\right)+(1-\lambda) / 3 \ln \left(\left(\sigma_{\alpha}\right)^{2} / \rho_{\alpha}\right)} .
\end{aligned}
$$

The expression of multiplicative accuracy function of $\widetilde{\alpha}$ can be further written as

$$
\begin{aligned}
A_{\lambda}(\widetilde{\alpha}) & =\left(\frac{\left(\rho_{\alpha}\right)^{(2 / 3)}}{\left(\sigma_{\alpha}\right)^{(1 / 3)}}\right)^{\lambda}\left(\frac{\left(\sigma_{\alpha}\right)^{(2 / 3)}}{\left(\rho_{\alpha}\right)^{(1 / 3)}}\right)^{1-\lambda} \\
& =\left(\sqrt[3]{\frac{\left(\rho_{\alpha}\right)^{2}}{\sigma_{\alpha}}}\right)^{\lambda}\left(\sqrt[3]{\frac{\left(\sigma_{\alpha}\right)^{2}}{\rho_{\alpha}}}\right)^{1-\lambda}
\end{aligned}
$$

which can be equivalently written as

$$
A_{\lambda}(\widetilde{\alpha})=\left(\frac{\rho_{\alpha}}{\sigma_{\alpha}}\right)^{\lambda} \cdot \frac{\left(\sigma_{\alpha}\right)^{(2 / 3)}}{\left(\rho_{\alpha}\right)^{(1 / 3)}}=\left(\frac{\rho_{\alpha}}{\sigma_{\alpha}}\right)^{\lambda} \cdot \sqrt[3]{\frac{\left(\sigma_{\alpha}\right)^{2}}{\rho_{\alpha}}} .
$$

The multiplicative accuracy function based on risk preference satisfies some desirable properties as follows.

Property 1. For any two intuitionistic multiplicative numbers $\widetilde{\alpha}_{1}=\left(\rho_{1}, \sigma_{1}\right)$ and $\widetilde{\alpha}_{2}=\left(\rho_{2}, \sigma_{2}\right), A_{\text {Xia }}\left(\widetilde{\alpha}_{1}\right)>A_{\text {Xia }}\left(\widetilde{\alpha}_{2}\right)$ if and only if $A_{0.5}\left(\widetilde{\alpha}_{1}\right)>A_{0.5}\left(\widetilde{\alpha}_{2}\right)$.

Proof. If $A_{\mathrm{Xia}}\left(\widetilde{\alpha}_{1}\right)>A_{\mathrm{Xia}}\left(\widetilde{\alpha}_{2}\right)$, by equation (3), we have $\rho_{1} \sigma_{1}>\rho_{2} \sigma_{2}$.

When $\lambda=0.5$, from (12), we have $A_{0.5}\left(\widetilde{\alpha}_{i}\right)=\left(\rho_{i} \sigma_{i}\right)^{(1 / 6)}$, $i=1,2$.

For $i=1,2$, it follows that $A_{\text {Xia }}\left(\widetilde{\alpha}_{i}\right)=\left(A_{0.5}\left(\widetilde{\alpha}_{i}\right)\right)^{6}$.

Therefore, $A_{\mathrm{Xia}}\left(\widetilde{\alpha}_{1}\right)>A_{\mathrm{Xia}}\left(\widetilde{\alpha}_{2}\right)$ is equivalently written as

$$
A_{0.5}\left(\widetilde{\alpha}_{1}\right)=\left(A_{\mathrm{Xia}}\left(\widetilde{\alpha}_{1}\right)\right)^{(1 / 6)}>\left(A_{\mathrm{Xia}}\left(\widetilde{\alpha}_{2}\right)\right)^{(1 / 6)}=A_{0.5}\left(\widetilde{\alpha}_{2}\right) \text {. }
$$

The proof of Property 1 is completed.

Property 2. Let $\tilde{\alpha}=\left(\rho_{\alpha}, \sigma_{\alpha}\right)$ be an intuitionistic multiplicative number. $\forall \lambda \in[0,1]$, we have

$$
\begin{aligned}
& \frac{1}{9} \leq \min \left\{\sqrt[3]{\frac{\left(\rho_{\alpha}\right)^{2}}{\sigma_{\alpha}}}, \sqrt[3]{\frac{\left(\sigma_{\alpha}\right)^{2}}{\rho_{\alpha}}}\right\} \\
& \leq A_{\lambda}(\widetilde{\alpha}) \leq \max \left\{\sqrt[3]{\frac{\left(\rho_{\alpha}\right)^{2}}{\sigma_{\alpha}}}, \sqrt[3]{\frac{\left(\sigma_{\alpha}\right)^{2}}{\rho_{\alpha}}}\right\} \leq 9 .
\end{aligned}
$$

Proof. We prove Property 1 in the following two cases:

(1) If $\rho_{\alpha} \geq \sigma_{\alpha}$, then we have $\sqrt[3]{\left(\left(\sigma_{\alpha}\right)^{2} / \rho_{\alpha}\right)} \leq \sqrt[3]{\left(\left(\rho_{\alpha}\right)^{2} / \sigma_{\alpha}\right)}$. $\forall \lambda \in[0,1]$, we have

$$
\sqrt[3]{\frac{\left(\sigma_{\alpha}\right)^{2}}{\rho_{\alpha}}} \leq\left(\sqrt[3]{\frac{\left(\rho_{\alpha}\right)^{2}}{\sigma_{\alpha}}}\right)^{\lambda}\left(\sqrt[3]{\frac{\left(\sigma_{\alpha}\right)^{2}}{\rho_{\alpha}}}\right)^{1-\lambda} \leq \sqrt[3]{\frac{\left(\rho_{\alpha}\right)^{2}}{\sigma_{\alpha}}} .
$$

Since $\rho_{\alpha}, \sigma_{\alpha} \in[(1 / 9), 9]$, we have

$$
\frac{1}{9} \leq \sqrt[3]{\frac{\left(\sigma_{\alpha}\right)^{2}}{\rho_{\alpha}}} \leq\left(\sqrt[3]{\frac{\left(\rho_{\alpha}\right)^{2}}{\sigma_{\alpha}}}\right)^{\lambda}\left(\sqrt[3]{\frac{\left(\sigma_{\alpha}\right)^{2}}{\rho_{\alpha}}}\right)^{1-\lambda} \leq \sqrt[3]{\frac{\left(\rho_{\alpha}\right)^{2}}{\sigma_{\alpha}}} \leq 9
$$

(2) If $\rho_{\alpha}<\sigma_{\alpha}$, then we have $\sqrt[3]{\left(\left(\rho_{\alpha}\right)^{2} / \sigma_{\alpha}\right)} \leq \sqrt[3]{\left(\left(\sigma_{\alpha}\right)^{2} / \rho_{\alpha}\right)}$. Since $\rho_{\alpha}, \sigma_{\alpha} \in[(1 / 9), 9]$, for all $\lambda \in[0,1]$, we have

$$
\frac{1}{9} \leq \sqrt[3]{\frac{\left(\rho_{\alpha}\right)^{2}}{\sigma_{\alpha}}} \leq\left(\sqrt[3]{\frac{\left(\rho_{\alpha}\right)^{2}}{\sigma_{\alpha}}}\right)^{\lambda}\left(\sqrt[3]{\frac{\left(\sigma_{\alpha}\right)^{2}}{\rho_{\alpha}}}\right)^{1-\lambda} \leq \sqrt[3]{\frac{\left(\sigma_{\alpha}\right)^{2}}{\rho_{\alpha}}} \leq 9 .
$$

Considering both cases (1) and (2), we have

$$
\begin{aligned}
\frac{1}{9} & \leq \min \left\{\sqrt[3]{\frac{\left(\rho_{\alpha}\right)^{2}}{\sigma_{\alpha}}}, \sqrt[3]{\frac{\left(\sigma_{\alpha}\right)^{2}}{\rho_{\alpha}}}\right\} \\
& \leq A_{\lambda}(\widetilde{\alpha}) \leq \max \left\{\sqrt[3]{\frac{\left(\rho_{\alpha}\right)^{2}}{\sigma_{\alpha}}}, \sqrt[3]{\frac{\left(\sigma_{\alpha}\right)^{2}}{\rho_{\alpha}}}\right\} \leq 9 .
\end{aligned}
$$

Therefore, Property 2 is proved.

Property 3. Let $\tilde{\alpha}_{1}=\left(\rho_{1}, \sigma_{1}\right)$ and $\tilde{\alpha}_{2}=\left(\rho_{2}, \sigma_{2}\right)$ be two intuitionistic multiplicative numbers. $\forall \lambda \in[0,1]$; if $\left(\sigma_{1} / \sigma_{2}\right) \leq\left(\rho_{1} / \rho_{2}\right) \leq\left(\sigma_{1} / \sigma_{2}\right)^{2}$, then $A_{\lambda}\left(\widetilde{\alpha}_{1}\right) \geq A_{\lambda}\left(\widetilde{\alpha}_{2}\right)$

Proof. Based on equation (7), we have

$$
\begin{aligned}
& A_{\lambda}\left(\widetilde{\alpha}_{1}\right)=\left(\frac{\rho_{1}}{\sigma_{1}}\right)^{\lambda} \cdot \frac{\left(\sigma_{1}\right)^{(2 / 3)}}{\left(\rho_{1}\right)^{(1 / 3)}}, \\
& A_{\lambda}\left(\widetilde{\alpha}_{2}\right)=\left(\frac{\rho_{2}}{\sigma_{2}}\right)^{\lambda} \cdot \frac{\left(\sigma_{2}\right)^{(2 / 3)}}{\left(\rho_{2}\right)^{(1 / 3)}} .
\end{aligned}
$$

$A_{\lambda}\left(\widetilde{\alpha}_{1}\right)$ divided by $A_{\lambda}\left(\widetilde{\alpha}_{2}\right)$, we have

$$
\frac{A_{\lambda}\left(\widetilde{\alpha}_{1}\right)}{A_{\lambda}\left(\widetilde{\alpha}_{2}\right)}=\left(\frac{\rho_{1} \sigma_{2}}{\rho_{2} \sigma_{1}}\right)^{\lambda} \cdot \frac{\left(\sigma_{1} / \sigma_{2}\right)^{(2 / 3)}}{\left(\rho_{1} / \rho_{2}\right)^{(1 / 3)}} .
$$

The above expression can be equivalently written as $\ln A_{\lambda}\left(\widetilde{\alpha}_{1}\right)-\ln A_{\lambda}\left(\widetilde{\alpha}_{2}\right)=\lambda\left(\ln \rho_{1}-\ln \rho_{2}+\ln \sigma_{2}-\ln \sigma_{1}\right)+$ $(1 / 3)\left(2 \ln \sigma_{1}-2 \ln \sigma_{2}-\ln \rho_{1}+\ln \rho_{2}\right)$. Since $\left(\sigma_{1} / \sigma_{2}\right) \leq\left(\rho_{1} /\right.$ $\left.\rho_{2}\right) \leq\left(\sigma_{1} / \sigma_{2}\right)^{2}$, we have $\ln \sigma_{1}-\ln \sigma_{2} \leq \ln \rho_{1}-\ln \rho_{2} \leq$ $2\left(\ln \sigma_{1}-\ln \sigma_{2}\right)$. Accordingly, for all $\lambda \in[0,1], \ln A_{\lambda}\left(\widetilde{\alpha}_{1}\right)-$ $\ln A_{\lambda}\left(\widetilde{\alpha}_{2}\right) \geq 0$ holds. Namely, we have $A_{\lambda}\left(\widetilde{\alpha}_{1}\right) \geq A_{\lambda}\left(\widetilde{\alpha}_{2}\right)$. Therefore, the proof of Property 3 is completed.

Property 4. Let $\Delta \lambda$ be a perturbation of parameter $\lambda$ with $\lambda+\Delta \lambda \in[0,1]$. Given two intuitionistic multiplicative numbers $\widetilde{\alpha}_{1}=\left(\rho_{1}, \sigma_{1}\right)$ and $\widetilde{\alpha}_{2}=\left(\rho_{2}, \sigma_{2}\right)$. If $A_{\lambda}\left(\widetilde{\alpha}_{1}\right) \leq A_{\lambda}\left(\widetilde{\alpha}_{2}\right)$, then $A_{\lambda+\Delta \lambda}\left(\widetilde{\alpha}_{1}\right) \leq A_{\lambda+\Delta \lambda}\left(\widetilde{\alpha}_{2}\right)$ holds if and only if 


$$
\left\{\begin{array}{l}
-\lambda \leq \Delta \lambda \leq 1-\lambda, \quad \rho_{1} \sigma_{2}=\rho_{2} \sigma_{1} \\
\max \left\{-\lambda, \frac{\ln A_{\lambda}\left(\widetilde{\alpha}_{2}\right)-\ln A_{\lambda}\left(\widetilde{\alpha}_{1}\right)}{\ln \rho_{1}-\ln \rho_{2}+\ln \sigma_{2}-\ln \sigma_{1}}\right\} \leq \Delta \lambda \leq 1-\lambda, \quad \rho_{1} \sigma_{2}<\rho_{2} \sigma_{1} \\
-\lambda \leq \Delta \lambda \leq \min \left\{1-\lambda, \frac{\ln A_{\lambda}\left(\widetilde{\alpha}_{2}\right)-\ln A_{\lambda}\left(\widetilde{\alpha}_{1}\right)}{\ln \rho_{1}-\ln \rho_{2}+\ln \sigma_{2}-\ln \sigma_{1}}\right\}, \quad \rho_{1} \sigma_{2}>\rho_{2} \sigma_{1} .
\end{array}\right.
$$

Proof. Since $0 \leq \lambda+\Delta \lambda \leq 1$, we have $-\lambda \leq \Delta \lambda \leq 1-\lambda$. By equation (12), it holds that

$$
\begin{aligned}
& A_{\lambda+\Delta \lambda}\left(\widetilde{\alpha}_{1}\right)=A_{\lambda}\left(\widetilde{\alpha}_{1}\right) \cdot\left(\frac{\rho_{1}}{\sigma_{1}}\right)^{\Delta \lambda}, \\
& A_{\lambda+\Delta \lambda}\left(\widetilde{\alpha}_{2}\right)=A_{\lambda}\left(\widetilde{\alpha}_{2}\right) \cdot\left(\frac{\rho_{2}}{\sigma_{2}}\right)^{\Delta \lambda} .
\end{aligned}
$$

Accordingly, the following two inequalities are equivalent:

$$
A_{\lambda+\Delta \lambda}\left(\widetilde{\alpha}_{1}\right) \leq A_{\lambda+\Delta \lambda}\left(\widetilde{\alpha}_{2}\right) \Longleftrightarrow \frac{A_{\lambda}\left(\widetilde{\alpha}_{1}\right)}{A_{\lambda}\left(\widetilde{\alpha}_{2}\right)} \leq\left(\frac{\rho_{2} \sigma_{1}}{\rho_{1} \sigma_{2}}\right)^{\Delta \lambda} .
$$

In the following, we analyze (23) in three cases:

(1) If $\rho_{1} \sigma_{2}=\rho_{2} \sigma_{1}$, then inequalities $A_{\lambda}\left(\widetilde{\alpha}_{1}\right) \leq A_{\lambda}\left(\widetilde{\alpha}_{2}\right)$ and $A_{\lambda+\Delta \lambda}\left(\widetilde{\alpha}_{1}\right) \leq A_{\lambda+\Delta \lambda}\left(\widetilde{\alpha}_{2}\right)$ are equivalent. Thus, $\forall$ $\Delta \lambda \in \in[-\lambda, 1-\lambda]$, and relational expression (12) is true.

(2) If $\rho_{1} \sigma_{2}<\rho_{2} \sigma_{1}$, then inequality $A_{\lambda+\Delta \lambda}\left(\widetilde{\alpha}_{1}\right) \leq A_{\lambda+\Delta \lambda}\left(\widetilde{\alpha}_{2}\right)$ is equivalent to $\Delta \lambda \geq\left(\left(\ln A_{\lambda}\left(\widetilde{\alpha}_{2}\right)-\ln A_{\lambda}\left(\widetilde{\alpha}_{1}\right)\right) /\right.$ $\left.\left(\ln \rho_{1}-\ln \rho_{2}+\ln \sigma_{2}-\ln \sigma_{1}\right)\right)$.

Considering $-\lambda \leq \Delta \lambda \leq 1-\lambda$, we have

$$
\begin{aligned}
A_{\lambda+\Delta \lambda}\left(\widetilde{\alpha}_{1}\right) \leq & A_{\lambda+\Delta \lambda}\left(\widetilde{\alpha}_{2}\right) \Longleftrightarrow \max \\
& \cdot\left\{-\lambda, \frac{\ln A_{\lambda}\left(\widetilde{\alpha}_{2}\right)-\ln A_{\lambda}\left(\widetilde{\alpha}_{1}\right)}{\ln \rho_{1}-\ln \rho_{2}+\ln \sigma_{2}-\ln \sigma_{1}}\right\}
\end{aligned}
$$

(3) If $\rho_{1} \sigma_{2}>\rho_{2} \sigma_{1}$, then inequality $A_{\lambda+\Delta \lambda}\left(\widetilde{\alpha}_{1}\right) \leq A_{\lambda+\Delta \lambda}\left(\widetilde{\alpha}_{2}\right)$ is equivalent to $\Delta \lambda \leq \quad\left(\left(\ln A_{\lambda}\left(\widetilde{\alpha}_{2}\right)-\ln A_{\lambda}\left(\widetilde{\alpha}_{1}\right)\right)\right.$ $\left./\left(\ln \rho_{1}-\ln \rho_{2}+\ln \sigma_{2}-\ln \sigma_{1}\right)\right)$.

Since $-\lambda \leq \Delta \lambda \leq 1-\lambda$, we have

$$
\begin{aligned}
A_{\lambda+\Delta \lambda}\left(\widetilde{\alpha}_{1}\right) & \leq A_{\lambda+\Delta \lambda}\left(\widetilde{\alpha}_{2}\right) \Longleftrightarrow-\lambda \leq \Delta \lambda \\
& \leq \min \left\{1-\lambda, \frac{\ln A_{\lambda}\left(\widetilde{\alpha}_{2}\right)-\ln A_{\lambda}\left(\widetilde{\alpha}_{1}\right)}{\ln \rho_{1}-\ln \rho_{2}+\ln \sigma_{2}-\ln \sigma_{1}}\right\} .
\end{aligned}
$$

In sum, the proof of Property 4 is completed.

\subsection{The Multiplicative Score Function Based on Risk} Preference

Definition 6. Let $\widetilde{\alpha}=\left(\rho_{\alpha}, \sigma_{\alpha}\right)$ be an intuitionistic multiplicative number. The multiplicative score function of $\widetilde{\alpha}$ based on risk preference is defined by

$$
S_{\lambda}(\widetilde{\alpha})=e^{\left(\iint_{R_{\alpha}}((1-\lambda) x-\lambda y) \mathrm{d} x \mathrm{~d} y /\left(M\left(R_{\alpha}\right)\right)\right),}
$$

where $R_{\alpha}$ is the mapping feasible region of $\widetilde{\alpha}$, and $M\left(R_{\alpha}\right)$ is the area of mapping feasible region $R_{\alpha}$. The parameter $\lambda$ reflects the risk tendency of the decision maker.

It clear that $S_{\lambda}(\widetilde{\alpha})$ represents the geometric average of score functions over all feasible values. According to the operational properties of double integral, we have

$$
\leq \Delta \lambda \leq 1-\lambda .
$$

$$
\begin{aligned}
\iint_{R_{\alpha}}((1-\lambda) x-\lambda y) \mathrm{d} x \mathrm{~d} y & =\int_{\bar{\rho}_{\alpha}}^{-\bar{\sigma}_{\alpha}}\left(\int_{\bar{\sigma}_{\alpha}}^{-x}((1-\lambda) x-\lambda y) \mathrm{d} y\right) \mathrm{d} x \\
& =\int_{\bar{\rho}_{\alpha}}^{-\bar{\sigma}_{\alpha}}\left(-\frac{2-\lambda}{2} x^{2}-(1-\lambda) \bar{\sigma}_{\alpha} x+\lambda \frac{\left(\bar{\sigma}_{\alpha}\right)^{2}}{2}\right) \mathrm{d} x \\
& =-\frac{\lambda+1}{6}\left(\bar{\sigma}_{\alpha}\right)^{3}+\frac{2-\lambda}{6}\left(\bar{\rho}_{\alpha}\right)^{3}+\frac{(1-\lambda) \bar{\sigma}_{\alpha}}{2}\left(\bar{\rho}_{\alpha}\right)^{2}-\frac{\lambda}{2}\left(\bar{\sigma}_{\alpha}\right)^{2} \bar{\rho}_{\alpha} .
\end{aligned}
$$


Based on (26), it follows that

$$
\begin{aligned}
S_{\lambda}(\widetilde{\alpha}) & =e^{\left(\iint_{R_{\alpha}}((1-\lambda) x-\lambda y) d x d y /\left(M\left(R_{\alpha}\right)\right)\right)} \\
& =e^{\left(\left(-2\left(\bar{\sigma}_{\alpha}\right)^{3}+\left(\bar{\rho}_{\alpha}\right)^{3}-3\left(\bar{\sigma}_{\alpha}\right)^{2} \bar{\rho}_{\alpha}+(1-\lambda)\left(\left(\bar{\rho}_{\alpha}\right)^{3}+\left(\bar{\sigma}_{\alpha}\right)^{3}+3\left(\bar{\rho}_{\alpha}\right)^{2} \bar{\sigma}_{\alpha}+3\left(\bar{\sigma}_{\alpha}\right)^{2} \bar{\rho}_{\alpha}\right)\right) /\left(3\left(\bar{\rho}_{\alpha}+\bar{\sigma}_{\alpha}\right)^{2}\right)\right)} \\
& =e^{\left(\left(\left(\bar{\rho}_{\alpha}-2 \bar{\sigma}_{\alpha}\right)\left(\bar{\sigma}_{\alpha}+\bar{\rho}_{\alpha}\right)^{2}+(1-\lambda)\left(\bar{\sigma}_{\alpha}+\bar{\rho}_{\alpha}\right)^{3}\right) /\left(3\left(\bar{\rho}_{\alpha}+\bar{\sigma}_{\alpha}\right)^{2}\right)\right)} \\
& =e^{(1 / 3)\left(\bar{\rho}_{\alpha}-2 \bar{\sigma}_{\alpha}\right)+((1-\lambda) / 3)\left(\bar{\rho}_{\alpha}+\bar{\sigma}_{\alpha}\right)}
\end{aligned}
$$

Since $\bar{\rho}_{\alpha}=\ln \rho_{\alpha}$ and $\bar{\sigma}_{\alpha}=\ln \sigma_{\alpha}$, we have

$$
\begin{aligned}
S_{\lambda}(\widetilde{\alpha}) & =e^{(1-\lambda)\left(\left(2 \ln \rho_{\alpha}-\ln \sigma_{\alpha}\right) / 3\right)+\lambda\left(\left(\ln \rho_{\alpha}-2 \ln \sigma_{\alpha}\right) / 3\right)} \\
& =e^{((1-\lambda) / 3) \ln \left(\left(\rho_{\alpha}\right)^{2} / \sigma_{\alpha}\right)+(\lambda / 3) \ln \left(\rho_{\alpha} /\left(\sigma_{\alpha}\right)^{2}\right)} .
\end{aligned}
$$

The expression of multiplicative score function of $\widetilde{\alpha}$ can be further written as

$$
\begin{aligned}
S_{\lambda}(\widetilde{\alpha}) & =\left(\frac{\left(\rho_{\alpha}\right)^{(2 / 3)}}{\left(\sigma_{\alpha}\right)^{(1 / 3)}}\right)^{1-\lambda}\left(\frac{\left(\rho_{\alpha}\right)^{(1 / 3)}}{\left(\sigma_{\alpha}\right)^{(2 / 3)}}\right)^{\lambda} \\
& =\left(\sqrt[3]{\frac{\rho_{\alpha}}{\left(\sigma_{\alpha}\right)^{2}}}\right)^{\lambda}\left(\sqrt[3]{\frac{\left(\rho_{\alpha}\right)^{2}}{\sigma_{\alpha}}}\right)^{1-\lambda},
\end{aligned}
$$

which can be equivalently written as

$$
S_{\lambda}(\widetilde{\alpha})=\left(\frac{1}{\sqrt[3]{\rho_{\alpha} \sigma_{\alpha}}}\right)^{\lambda} \cdot \sqrt[3]{\frac{\left(\rho_{\alpha}\right)^{2}}{\sigma_{\alpha}}}
$$

The multiplicative score function based on risk preference satisfies some desirable properties as follows.

Property 5. Let $\widetilde{\alpha}=\left(\rho_{\alpha}, \sigma_{\alpha}\right)$ be an intuitionistic multiplicative number. $\forall \lambda_{1}, \lambda_{2} \in[0,1] ; \quad$ if $\lambda_{1} \leq \lambda_{2}$, then $S_{\lambda_{1}}(\widetilde{\alpha}) \leq S_{\lambda_{2}}(\widetilde{\alpha})$.

Proof. Based on equation (31), we have

$$
\begin{aligned}
& S_{\lambda_{1}}(\widetilde{\alpha})=\left(\frac{1}{\sqrt[3]{\rho_{\alpha} \sigma_{\alpha}}}\right)^{\lambda_{1}} \cdot \sqrt[3]{\frac{\left(\rho_{\alpha}\right)^{2}}{\sigma_{\alpha}}}, \\
& S_{\lambda_{2}}(\widetilde{\alpha})=\left(\frac{1}{\sqrt[3]{\rho_{\alpha} \sigma_{\alpha}}}\right)^{\lambda_{2}} \cdot \sqrt[3]{\frac{\left(\rho_{\alpha}\right)^{2}}{\sigma_{\alpha}}} .
\end{aligned}
$$

It follows that

$$
\frac{S_{\lambda_{1}}(\widetilde{\alpha})}{S_{\lambda_{2}}(\widetilde{\alpha})}=\frac{\left(1 /\left(\sqrt[3]{\rho_{\alpha} \sigma_{\alpha}}\right)\right)^{\lambda_{1}} \cdot \sqrt[3]{\left(\left(\rho_{\alpha}\right)^{2} / \sigma_{\alpha}\right)}}{\left(1 /\left(\sqrt[3]{\rho_{\alpha} \sigma_{\alpha}}\right)\right)^{\lambda_{2}} \cdot \sqrt[3]{\left(\left(\rho_{\alpha}\right)^{2} / \sigma_{\alpha}\right)}}=\left(\sqrt[3]{\rho_{\alpha} \sigma_{\alpha}}\right)^{\lambda_{2}-\lambda_{1}} .
$$

Since $0<\rho_{\alpha} \sigma_{\alpha} \leq 1$ and $\lambda_{2}-\lambda_{1} \geq 0, \quad$ we have $S_{\lambda_{1}}(\widetilde{\alpha}) \leq S_{\lambda_{2}}(\widetilde{\alpha})$.

The proof of Property 5 is completed.

Property 6. For any two intuitionistic multiplicative numbers $\widetilde{\alpha}_{1}=\left(\rho_{1}, \sigma_{1}\right)$ and $\widetilde{\alpha}_{2}=\left(\rho_{2}, \sigma_{2}\right), S_{\mathrm{Xia}}\left(\widetilde{\alpha}_{1}\right)>S_{\mathrm{Xia}}\left(\widetilde{\alpha}_{2}\right)$ if and only if $S_{0.5}\left(\widetilde{\alpha}_{1}\right)>S_{0.5}\left(\widetilde{\alpha}_{2}\right)$.

Proof:. If $S_{\mathrm{Xia}}\left(\widetilde{\alpha}_{1}\right)>S_{\mathrm{Xia}}\left(\widetilde{\alpha}_{2}\right)$, by equation (2), we have $\left(\rho_{1} / \sigma_{1}\right)>\left(\rho_{2} / \sigma_{2}\right)$.

When $\lambda=0.5$, from (31), we have $S_{0.5}\left(\widetilde{\alpha}_{i}\right)=\left(\rho_{i} / \sigma_{i}\right)^{(1 / 2)}$, $i=1,2$.

For $i=1,2$, it holds that $S_{\mathrm{Xia}}\left(\widetilde{\alpha}_{i}\right)=\left(S_{0.5}\left(\widetilde{\alpha}_{i}\right)\right)^{2}$.

Therefore, $S_{\mathrm{Xia}}\left(\widetilde{\alpha}_{1}\right)>S_{\mathrm{Xia}}\left(\widetilde{\alpha}_{2}\right)$ is equivalently written as

$$
S_{0.5}\left(\widetilde{\alpha}_{1}\right)=\left(S_{\mathrm{Xia}}\left(\widetilde{\alpha}_{1}\right)\right)^{(1 / 2)}>\left(S_{\mathrm{Xia}}\left(\widetilde{\alpha}_{2}\right)\right)^{(1 / 2)}=S_{0.5}\left(\widetilde{\alpha}_{2}\right) \text {. }
$$

The proof of Property 6 is completed.

Property 7. Let $\widetilde{\alpha}=\left(\rho_{\alpha}, \sigma_{\alpha}\right)$ be an intuitionistic multiplicative number. $\forall \lambda \in[0,1]$, we have $(1 / 9) \leq$ $\sqrt[3]{\left(\left(\rho_{\alpha}\right)^{2} / \sigma_{\alpha}\right)} \leq S_{\lambda}(\widetilde{\alpha}) \leq \sqrt[3]{\left(\rho_{\alpha} /\left(\sigma_{\alpha}\right)^{2}\right)} \leq 9$.

Proof. From equation (31), we have $S_{0}(\widetilde{\alpha})=\sqrt[3]{\left(\left(\rho_{\alpha}\right)^{2} / \sigma_{\alpha}\right)}$ and $S_{1}(\widetilde{\alpha})=\sqrt[3]{\left(\rho_{\alpha} /\left(\sigma_{\alpha}\right)^{2}\right)}$. By Property 5 , for all $\lambda \in[0,1]$, we have $S_{0}(\widetilde{\alpha}) \leq S_{\lambda}(\widetilde{\alpha}) \leq S_{1}(\widetilde{\alpha})$. Since $\rho_{\alpha}(x), \sigma_{\alpha}(x) \in[(1 / 9), 9]$, we have

$$
\begin{aligned}
& S_{\lambda}(\widetilde{\alpha}) \geq S_{0}(\widetilde{\alpha})=\sqrt[3]{\frac{\left(\rho_{\alpha}\right)^{2}}{\sigma_{\alpha}}} \geq \frac{1}{9} \\
& S_{\lambda}(\widetilde{\alpha}) \leq S_{1}(\widetilde{\alpha})=\sqrt[3]{\frac{\rho_{\alpha}}{\left(\sigma_{\alpha}\right)^{2}}} \leq 9 .
\end{aligned}
$$

Therefore, Property 7 is proved.

Property 8. Let $\Delta \lambda$ be a perturbation of parameter $\lambda$ with $\lambda+\Delta \lambda \in[0,1]$. Given two intuitionistic multiplicative numbers $\widetilde{\alpha}_{1}=\left(\rho_{1}, \sigma_{1}\right)$ and $\widetilde{\alpha}_{2}=\left(\rho_{2}, \sigma_{2}\right)$. If $S_{\lambda}\left(\widetilde{\alpha}_{1}\right) \leq S_{\lambda}\left(\widetilde{\alpha}_{2}\right)$, then $S_{\lambda+\Delta \lambda}\left(\widetilde{\alpha}_{1}\right) \leq S_{\lambda+\Delta \lambda}\left(\widetilde{\alpha}_{2}\right)$ holds if and only if 


$$
\left\{\begin{array}{l}
-\lambda \leq \Delta \lambda \leq 1-\lambda, \quad \rho_{1} \sigma_{1}=\rho_{2} \sigma_{2}, \\
\max \left\{-\lambda, \frac{3 \ln S_{\lambda}\left(\widetilde{\alpha}_{2}\right)-3 \ln S_{\lambda}\left(\widetilde{\alpha}_{1}\right)}{\left.\ln \rho_{2}-\ln \rho_{1}+\ln \sigma_{2}-\ln \sigma_{1}\right\} \leq \Delta \lambda \leq 1-\lambda, \quad \rho_{1} \sigma_{1}>\rho_{2} \sigma_{2},}\right. \\
-\lambda \leq \Delta \lambda \leq \min \left\{1-\lambda, \frac{3 \ln S_{\lambda}\left(\widetilde{\alpha}_{2}\right)-3 \ln S_{\lambda}\left(\widetilde{\alpha}_{1}\right)}{\left.\ln \rho_{2}-\ln \rho_{1}+\ln \sigma_{2}-\ln \sigma_{1}\right\},} \quad \rho_{1} \sigma_{1}<\rho_{2} \sigma_{2} .\right.
\end{array}\right.
$$

Proof. Since $0 \leq \lambda+\Delta \lambda \leq 1$, we have $-\lambda \leq \Delta \lambda \leq 1-\lambda$. By equation (31), it holds that

$$
\begin{aligned}
& S_{\lambda+\Delta \lambda}\left(\widetilde{\alpha}_{1}\right)=S_{\lambda}\left(\widetilde{\alpha}_{1}\right) \cdot\left(\frac{1}{\sqrt[3]{\rho_{1} \sigma_{1}}}\right)^{\Delta \lambda}, \\
& S_{\lambda+\Delta \lambda}\left(\widetilde{\alpha}_{2}\right)=S_{\lambda}\left(\widetilde{\alpha}_{2}\right) \cdot\left(\frac{1}{\sqrt[3]{\rho_{2} \sigma_{2}}}\right)^{\Delta \lambda} .
\end{aligned}
$$

Accordingly, the following two inequalities are equivalent:

$$
S_{\lambda+\Delta \lambda}\left(\widetilde{\alpha}_{1}\right) \leq S_{\lambda+\Delta \lambda}\left(\widetilde{\alpha}_{2}\right) \Longleftrightarrow \frac{S_{\lambda}\left(\widetilde{\alpha}_{2}\right)}{S_{\lambda}\left(\widetilde{\alpha}_{1}\right)} \geq\left(\sqrt[3]{\frac{\rho_{2} \sigma_{2}}{\rho_{1} \sigma_{1}}}\right)^{\Delta \lambda} .
$$

In the following, we analyze (38) in three cases:

(1) If $\rho_{1} \sigma_{1}=\rho_{2} \sigma_{2}$, then inequalities $S_{\lambda}\left(\widetilde{\alpha}_{1}\right) \leq S_{\lambda}\left(\widetilde{\alpha}_{2}\right)$ and $S_{\lambda+\Delta \lambda}\left(\widetilde{\alpha}_{1}\right) \leq S_{\lambda+\Delta \lambda}\left(\widetilde{\alpha}_{2}\right)$ are equivalent. Thus, $\forall$ $\Delta \lambda \in \in[-\lambda, 1-\lambda]$, and the relational expression (38) is true.

(2) If $\rho_{1} \sigma_{1}>\rho_{2} \sigma_{2}$, then inequality $S_{\lambda+\Delta \lambda}\left(\widetilde{\alpha}_{1}\right) \leq S_{\lambda+\Delta \lambda}\left(\widetilde{\alpha}_{2}\right)$ is equivalent to $\Delta \lambda \geq\left(\left(3 \ln S_{\lambda}\left(\widetilde{\alpha}_{2}\right)-3 \ln S_{\lambda}\left(\widetilde{\alpha}_{1}\right)\right)\right.$ $\left./\left(\ln \rho_{2}-\ln \rho_{1}+\ln \sigma_{2}-\ln \sigma_{1}\right)\right)$.

Considering $-\lambda \leq \Delta \lambda \leq 1-\lambda$, we have

$$
\begin{aligned}
S_{\lambda+\Delta \lambda}\left(\widetilde{\alpha}_{1}\right) \leq & S_{\lambda+\Delta \lambda}\left(\widetilde{\alpha}_{2}\right) \Longleftrightarrow \max \\
& \cdot\left\{-\lambda, \frac{3 \ln S_{\lambda}\left(\widetilde{\alpha}_{2}\right)-3 \ln S_{\lambda}\left(\widetilde{\alpha}_{1}\right)}{\ln \rho_{2}-\ln \rho_{1}+\ln \sigma_{2}-\ln \sigma_{1}}\right\} \\
\leq & \Delta \lambda \leq 1-\lambda .
\end{aligned}
$$

(3) If $\rho_{1} \sigma_{1}<\rho_{2} \sigma_{2}$, then inequality $S_{\lambda+\Delta \lambda}\left(\widetilde{\alpha}_{1}\right) \leq S_{\lambda+\Delta \lambda}\left(\widetilde{\alpha}_{2}\right)$ is equivalent to $\Delta \lambda \leq\left(\left(3 \ln S_{\lambda}\left(\widetilde{\alpha}_{2}\right)-3 \ln S_{\lambda}\left(\widetilde{\alpha}_{1}\right)\right)\right.$ $\left./\left(\ln \rho_{2}-\ln \rho_{1}+\ln \sigma_{2}-\ln \sigma_{1}\right)\right)$.

Since $-\lambda \leq \Delta \lambda \leq 1-\lambda$, we have

$$
\begin{aligned}
S_{\lambda+\Delta \lambda}\left(\widetilde{\alpha}_{1}\right) & \leq S_{\lambda+\Delta \lambda}\left(\widetilde{\alpha}_{2}\right) \Longleftrightarrow-\lambda \leq \Delta \lambda \\
& \leq \min \left\{1-\lambda, \frac{3 \ln S_{\lambda}\left(\widetilde{\alpha}_{2}\right)-3 \ln S_{\lambda}\left(\widetilde{\alpha}_{1}\right)}{\ln \rho_{2}-\ln \rho_{1}+\ln \sigma_{2}-\ln \sigma_{1}}\right\} .
\end{aligned}
$$

In sum, the proof of Property 8 is completed.
Property 9. Let $\widetilde{\alpha}_{1}=\left(\rho_{1}, \sigma_{1}\right)$ and $\widetilde{\alpha}_{2}=\left(\rho_{2}, \sigma_{2}\right)$ be two intuitionistic multiplicative numbers. If $\rho_{1} \geq \rho_{2}$ and $\sigma_{1} \leq \sigma_{2}$, then for all $\lambda \in[0,1]$, we have $S_{\lambda}\left(\widetilde{\alpha}_{1}\right) \geq S_{\lambda}\left(\widetilde{\alpha}_{2}\right)$.

Proof. From equation (31), we have

$$
\begin{aligned}
& S_{\lambda}\left(\widetilde{\alpha}_{1}\right)=\left(\frac{1}{\sqrt[3]{\rho_{1} \sigma_{1}}}\right)^{\lambda} \cdot \sqrt[3]{\frac{\left(\rho_{1}\right)^{2}}{\sigma_{1}}}, \\
& S_{\lambda}\left(\widetilde{\alpha}_{2}\right)=\left(\frac{1}{\sqrt[3]{\rho_{2} \sigma_{2}}}\right)^{\lambda} \cdot \sqrt[3]{\frac{\left(\rho_{2}\right)^{2}}{\sigma_{2}}} .
\end{aligned}
$$

It follows that

$$
\begin{aligned}
\frac{S_{\lambda}\left(\widetilde{\alpha}_{1}\right)}{S_{\lambda}\left(\widetilde{\alpha}_{2}\right)} & =\frac{\left(1 /\left(\sqrt[3]{\rho_{1} \sigma_{1}}\right)\right)^{\lambda} \cdot \sqrt[3]{\left(\left(\rho_{1}\right)^{2} / \sigma_{1}\right)}}{\left(1 /\left(\sqrt[3]{\rho_{2} \sigma_{2}}\right)\right)^{\lambda} \cdot \sqrt[3]{\left(\left(\rho_{2}\right)^{2} / \sigma_{2}\right)}} \\
& =\left(\sqrt{\frac{\left(\sigma_{2}\right)^{2}}{\left(\sigma_{1}\right)^{2}} \frac{\rho_{1}}{\rho_{2}}}\right)^{\lambda}\left(\sqrt[\beta]{\frac{\left(\rho_{1}\right)^{2}}{\left(\rho_{2}\right)^{2}} \frac{\sigma_{2}}{\sigma_{1}}}\right)^{1-\lambda} .
\end{aligned}
$$

Since $\rho_{1} \geq \rho_{2}$ and $\sigma_{1} \leq \sigma_{2}$, we have $\left(\rho_{1} / \rho_{2}\right) \geq 1$ and $\left(\sigma_{2} / \sigma_{1}\right) \geq 1$. Considering $0 \leq \lambda \leq 1$, we have $S_{\lambda}\left(\widetilde{\alpha}_{1}\right) \geq S_{\lambda}\left(\widetilde{\alpha}_{2}\right)$.

Therefore, the proof of Property 9 is completed.

\section{The Order Relation between Intuitionistic Multiplicative Numbers}

In the following, the order relation between any two intuitionistic multiplicative numbers is defined based on the multiplicative score function and accuracy function.

Property 10. Let $\widetilde{\alpha}_{1}=\left(\rho_{1}, \sigma_{1}\right)$ and $\widetilde{\alpha}_{2}=\left(\rho_{2}, \sigma_{2}\right)$ be two intuitionistic multiplicative numbers. For each $\lambda \in[0,1]$, $\widetilde{\alpha}_{1}=\widetilde{\alpha}_{2}$ if and only if $A_{\lambda}\left(\widetilde{\alpha}_{1}\right)=A_{\lambda}\left(\widetilde{\alpha}_{2}\right)$ and $S_{\lambda}\left(\widetilde{\alpha}_{1}\right)=S_{\lambda}\left(\widetilde{\alpha}_{2}\right)$ hold.

Proof

(1) If $\widetilde{\alpha}_{1}=\widetilde{\alpha}_{2}$, then we have $\rho_{1}=\rho_{2}$ and $\sigma_{1}=\sigma_{2}$. Accordingly, $S_{\lambda}\left(\widetilde{\alpha}_{1}\right)=S_{\lambda}\left(\widetilde{\alpha}_{2}\right)$ and $A_{\lambda}\left(\widetilde{\alpha}_{1}\right)=A_{\lambda}\left(\widetilde{\alpha}_{2}\right)$ hold.

(2) For all $\lambda \in[0,1]$, if $S_{\lambda}\left(\widetilde{\alpha}_{1}\right)=S_{\lambda}\left(\widetilde{\alpha}_{2}\right) \quad$ and $A_{\lambda}\left(\widetilde{\alpha}_{1}\right)=A_{\lambda}\left(\widetilde{\alpha}_{2}\right)$, we have 


$$
\begin{gathered}
\left(\rho_{1} \sigma_{1}\right)^{-(\lambda / 3)} \cdot \frac{\left(\rho_{1}\right)^{(2 / 3)}}{\sigma_{1}^{(1 / 3)}}=\left(\rho_{2} \sigma_{2}\right)^{-(\lambda / 3)} \cdot \frac{\left(\rho_{2}\right)^{(2 / 3)}}{\sigma_{2}^{(1 / 3)}} \\
\left(\frac{\rho_{1}}{\sigma_{1}}\right)^{\lambda} \cdot \frac{\left(\sigma_{1}\right)^{(2 / 3)}}{\left(\rho_{1}\right)^{(1 / 3)}}=\left(\frac{\rho_{2}}{\sigma_{2}}\right)^{\lambda} \cdot \frac{\left(\sigma_{2}\right)^{(2 / 3)}}{\left(\rho_{2}\right)^{(1 / 3)}}
\end{gathered}
$$

From (43), we have

$(2-\lambda)\left(\ln \rho_{1}-\ln \rho_{2}\right)=(1+\lambda)\left(\ln \sigma_{1}-\ln \sigma_{2}\right)$.

By (44), we have

$(3 \lambda-1)\left(\ln \rho_{1}-\ln \rho_{2}\right)=(3 \lambda-2)\left(\ln \sigma_{1}-\ln \sigma_{2}\right)$,

(45) can be further written as

$$
\ln \rho_{1}-\ln \rho_{2}=\frac{1+\lambda}{2-\lambda} \cdot\left(\ln \sigma_{1}-\ln \sigma_{2}\right) .
$$

Applying equation (47) to (46), it follows that

$$
\begin{aligned}
& (3 \lambda-1)(1+\lambda)\left(\ln \sigma_{1}-\ln \sigma_{2}\right) \\
& =(2-\lambda)(3 \lambda-2)\left(\ln \sigma_{1}-\ln \sigma_{2}\right) .
\end{aligned}
$$

Obviously, we can derive from $\left(2 \lambda^{2}-2 \lambda+1\right)\left(\ln \sigma_{1}-\ln \sigma_{2}\right)=0$

(48) that

Since $2 \lambda^{2}-2 \lambda+1>0$ always holds, we have $\sigma_{1}=\sigma_{2}$. Based on (47), we have $\rho_{1}=\rho_{2}$. Therefore, it concludes that $\widetilde{\alpha}_{1}=\widetilde{\alpha}_{2}$.

In sum, the proof of Property 10 is completed

In the following, the order relation between any two intuitionistic multiplicative numbers is defined

Definition 7. Let $\widetilde{\alpha}_{1}=\left(\rho_{1}, \sigma_{1}\right)$ and $\widetilde{\alpha}_{2}=\left(\rho_{2}, \sigma_{2}\right)$ be two intuitionistic multiplicative numbers. For each $\lambda \in[0,1]$, if one of the following conditions is true,

(1) $S_{\lambda}\left(\widetilde{\alpha}_{1}\right)>S_{\lambda}\left(\widetilde{\alpha}_{2}\right)$

(2) $S_{\lambda}\left(\widetilde{\alpha}_{1}\right)=S_{\lambda}\left(\widetilde{\alpha}_{2}\right)$ and $A_{\lambda}\left(\widetilde{\alpha}_{1}\right)>A_{\lambda}\left(\widetilde{\alpha}_{2}\right)$

then $\widetilde{\alpha}_{1}$ is said to be superior to $\widetilde{\alpha}_{2}$, denoted by $\widetilde{\alpha}_{1}>_{\lambda} \widetilde{\alpha}_{2}$.

Property 11. Let $\widetilde{\alpha}_{1}, \widetilde{\alpha}_{2}$, and

$\widetilde{\alpha}_{3}$ be three intuitionistic multiplicative numbers. The order relation $>_{\lambda}$ in Definition 7 satisfies the following conditions:

(1) If $\widetilde{\alpha}_{2}>_{\lambda} \widetilde{\alpha}_{1}$, then $\widetilde{\alpha}_{1}>_{\lambda} \widetilde{\alpha}_{2}$ does not hold

(2) For each intuitionistic multiplicative number, $\widetilde{\alpha}_{1}$, $\widetilde{\alpha}_{1}>_{\lambda} \widetilde{\alpha}_{1}$ does not hold

(3) If $\widetilde{\alpha}_{1}>_{\lambda} \widetilde{\alpha}_{2}$ and $\widetilde{\alpha}_{2}>_{\lambda} \widetilde{\alpha}_{3}$, then $\widetilde{\alpha}_{1}>_{\lambda} \widetilde{\alpha}_{3}$

\section{Proof}

(1) Since $\widetilde{\alpha}_{2} \succ_{\lambda} \widetilde{\alpha}_{1}$, from Definition 7 , the results can be analyzed in the following two cases. When $S_{\lambda}\left(\widetilde{\alpha}_{2}\right)>S_{\lambda}\left(\widetilde{\alpha}_{1}\right)$, then $\widetilde{\alpha}_{1}>{ }_{\lambda} \widetilde{\alpha}_{2}$ does not hold. When
$S_{\lambda}\left(\widetilde{\alpha}_{1}\right)=S_{\lambda}\left(\widetilde{\alpha}_{2}\right)$ and $A_{\lambda}\left(\widetilde{\alpha}_{2}\right)>A_{\lambda}\left(\widetilde{\alpha}_{1}\right)$, then $\widetilde{\alpha}_{1}>_{\lambda} \widetilde{\alpha}_{2}$ still holds.

(2) If $\widetilde{\alpha}_{1}>_{\lambda} \widetilde{\alpha}_{1}$ holds, then from Definition 7, we have $S_{\lambda}\left(\widetilde{\alpha}_{1}\right)>S_{\lambda}\left(\widetilde{\alpha}_{1}\right)$ or $A_{\lambda}\left(\widetilde{\alpha}_{1}\right)>A_{\lambda}\left(\widetilde{\alpha}_{1}\right)$, which lead to the illogical results. Thus, for each intuitionistic multiplicative number, $\widetilde{\alpha}_{1}, \widetilde{\alpha}_{1}>_{\lambda} \widetilde{\alpha}_{1}$ does not hold.

(3) Since $\widetilde{\alpha}_{1} \succ_{\lambda} \widetilde{\alpha}_{2}$, the results can be considered in two cases:

(a) $S_{\lambda}\left(\widetilde{\alpha}_{1}\right)>S_{\lambda}\left(\widetilde{\alpha}_{2}\right)$. Because $\widetilde{\alpha}_{2}>_{\lambda} \widetilde{\alpha}_{3}$, from Definition 7 , we have $S_{\lambda}\left(\widetilde{\alpha}_{2}\right) \geq S_{\lambda}\left(\widetilde{\alpha}_{3}\right)$. Thus, we get $S_{\lambda}\left(\widetilde{\alpha}_{1}\right)>S_{\lambda}\left(\widetilde{\alpha}_{3}\right)$. It follows that $\widetilde{\alpha}_{1}>_{\lambda} \widetilde{\alpha}_{3}$.

(b) $S_{\lambda}\left(\widetilde{\alpha}_{1}\right)=S_{\lambda}\left(\widetilde{\alpha}_{2}\right)$ and $A_{\lambda}\left(\widetilde{\alpha}_{1}\right)>A_{\lambda}\left(\widetilde{\alpha}_{2}\right)$. Because $\tilde{\alpha}_{2}>_{\lambda} \widetilde{\alpha}_{3}$, we have

(i) If $S_{\lambda}\left(\widetilde{\alpha}_{2}\right)>S_{\lambda}\left(\widetilde{\alpha}_{3}\right)$, then $S_{\lambda}\left(\widetilde{\alpha}_{1}\right)>S_{\lambda}\left(\widetilde{\alpha}_{3}\right)$. Namely, we have $\widetilde{\alpha}_{1}>_{\lambda} \widetilde{\alpha}_{3}$.

(ii) If $S_{\lambda}\left(\widetilde{\alpha}_{2}\right)=S_{\lambda}\left(\widetilde{\alpha}_{3}\right)$ and $A_{\lambda}\left(\widetilde{\alpha}_{2}\right)>A_{\lambda}\left(\widetilde{\alpha}_{3}\right)$, then $S_{\lambda}\left(\widetilde{\alpha}_{1}\right)=S_{\lambda}\left(\widetilde{\alpha}_{3}\right)$ and $A_{\lambda}\left(\widetilde{\alpha}_{1}\right)>A_{\lambda}\left(\widetilde{\alpha}_{3}\right)$. From Definition 7 , we have $\widetilde{\alpha}_{1}>_{\lambda} \widetilde{\alpha}_{3}$.

In sum, the proof of Property 11 is complete.

It is clear that the order relation $>_{\lambda}$ on the set of intuitionistic multiplicative numbers is a strict order. The frame diagram of the proposed ranking method is illustrated in Figure 2.

Accordingly, the proposed multiplicative accuracy and score functions can be used to compare any two intuitionistic multiplicative numbers. By equations (2), (3), (12), and (31), we have $S_{0.5}(\widetilde{\alpha})=\left(\rho_{\alpha} \sigma_{\alpha}\right)^{(1 / 2)}=\left(S_{\text {Xia }}(\widetilde{\alpha})\right)^{(1 / 2)}$ and $A_{0.5}(\widetilde{\alpha})=\left(\rho_{\alpha} \sigma_{\alpha}\right)^{(1 / 6)}=\left(A_{\mathrm{Xia}}(\widetilde{\alpha})\right)^{(1 / 6)}$. It means that the total order relation defined in Definition 7 is equivalent to Xia's method [16] when $\lambda=0.5$. Clearly, Xia's ranking method is a special case of the proposed method based on multiplicative accuracy and score functions.

\section{Numerical Example and Comparison}

In the following, we apply the proposed score and accuracy functions to select the optimal logistics transfer station. In addition, a comparison example is developed to highlight the advantage and effectiveness of the proposed ranking method.

5.1. Application of the Multiplicative Score and Accuracy Functions in Selecting Logistics Transfer Station. With the increasingly frequent interaction of materials, the pressure of logistics enterprises is becoming more and more serious. It is necessary to setup some logistics transfer stations to make the material circulation process smoother. The optimal location of the transfer station can maximize the transportation efficiency of logistics enterprises. Suppose there is a panel with three logistics transfer stations: $\mathrm{TS}_{1}$ (transfer station I), $\mathrm{TS}_{2}$ (transfer station II), and $\mathrm{TS}_{3}$ (transfer station III). Logistics enterprise expects to select the optimal location of logistics transfer station according to four attributes: $B_{1}$ (traffic conditions), $B_{2}$ (built-up area), $B_{3}$ (number of surrounding residents), and $B_{4}$ (local economic 


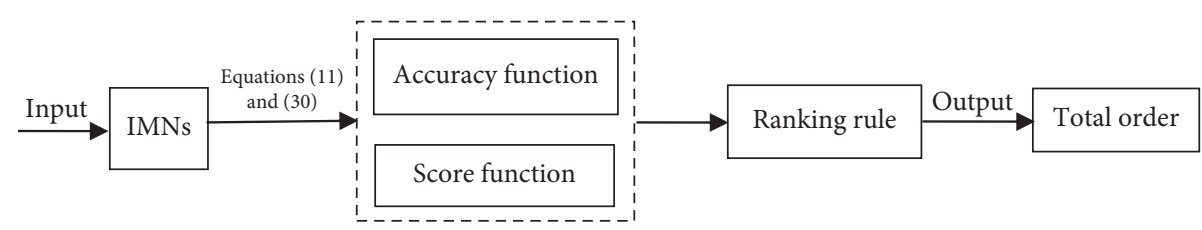

FIgure 2: The frame diagram of the proposed ranking method.

developing level). The weighting vector corresponding to attributes is $\omega=\left(\omega_{1}, \omega_{2}, \omega_{3}, \omega_{4}\right)=(0.1,0.4,0.3,0.2)$.

Step 1. Assume that three logistics transfer stations $\mathrm{TS}_{1}, \mathrm{TS}_{2}$, and $\mathrm{TS}_{3}$ are evaluated by intuitionistic multiplicative numbers under four attributes $B_{1} B_{2}, B_{3}$, and $B_{4}$. Accordingly, the intuitionistic multiplicative decision matrix $\widetilde{D}=$ $\left(\vec{d}_{i j}\right)_{3 \times 4}$ is obtained as

$$
\begin{aligned}
& \begin{array}{llll}
B_{1} & B_{2} & B_{3} & B_{4}
\end{array}
\end{aligned}
$$

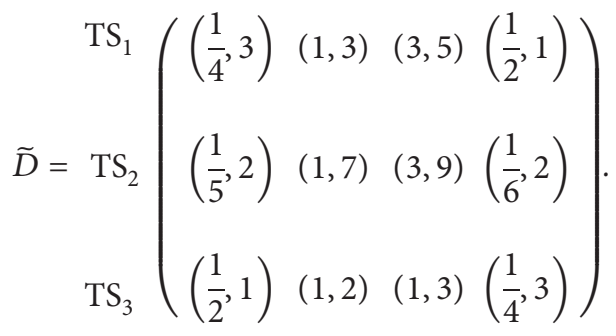

Step 2. By equation (31), the multiplicative score matrix $S_{\lambda}(\widetilde{D})=\left(S_{\lambda}\left(d_{i j}\right)\right)_{3 \times 4}$ with respect to parameter $\lambda$ is derived as follows:

$$
S_{\lambda}(\widetilde{D})=\left(\begin{array}{llll}
0.275 \cdot 1.101^{\lambda} & 0.693 \cdot 0.693^{\lambda} & 1.216 \cdot 0.405^{\lambda} & 0.630 \cdot 1.260^{\lambda} \\
0.271 \cdot 1.357^{\lambda} & 0.523 \cdot 0.523^{\lambda} & 1.000 \cdot 0.333^{\lambda} & 0.240 \cdot 1.442^{\lambda} \\
0.630 \cdot 1.260^{\lambda} & 0.794 \cdot 0.794^{\lambda} & 0.693 \cdot 0.693^{\lambda} & 0.275 \cdot 1.101^{\lambda}
\end{array}\right) .
$$

Step 3. The overall multiplicative score values of alternatives $\mathrm{TS}_{1}, \mathrm{TS}_{2}$, and $\mathrm{TS}_{3}$ are calculated as follows:

$$
\begin{aligned}
& \mathrm{OS}\left(\mathrm{TS}_{1}, \lambda\right)=\prod_{j=1}^{4} s_{\lambda}\left(\tilde{d}_{1 j}\right)^{\omega_{j}}=0.734 \cdot 0.696^{\lambda}, \\
& \mathrm{OS}\left(\mathrm{TS}_{2}, \lambda\right)=\prod_{j=1}^{4} s_{\lambda}\left(\tilde{d}_{2 j}\right)^{\omega_{j}}=0.509 \cdot 0.615^{\lambda}, \\
& \mathrm{OS}\left(\mathrm{TS}_{3}, \lambda\right)=\prod_{j=1}^{4} s_{\lambda}\left(\tilde{d}_{3 j}\right)^{\omega_{j}}=0.602 \cdot 0.852^{\lambda} .
\end{aligned}
$$

For parameter $\lambda \in[0,1]$, the curves corresponding to three overall multiplicative score values are illustrated in Figure 3.

Step 4. It is clear that $\operatorname{OS}\left(\mathrm{TS}_{1}, \lambda\right)>\operatorname{OS}\left(\mathrm{TS}_{2}, \lambda\right)$ and OS $\left(\mathrm{TS}_{3}, \lambda\right)>\mathrm{OS}\left(\mathrm{TS}_{2}, \lambda\right)$ hold for all $\lambda \in[0,1]$. Since $\left(\left(\mathrm{OS}\left(\mathrm{TS}_{1}, \lambda\right)\right) /\left(\mathrm{OS}\left(\mathrm{TS}_{3}, \lambda\right)\right)\right)=1.219 \cdot 0.817^{\lambda}$, we consider three cases as follows:

(1) If $\lambda \in[0,0.98)$, then we have $\operatorname{OS}\left(\mathrm{TS}_{1}, \lambda\right)>$ $\mathrm{OS}\left(\mathrm{TS}_{3}, \lambda\right)$, i.e., $\mathrm{TS}_{1}>\mathrm{TS}_{3}$
(2) If $\lambda \in(0.98,1]$, then we have $\operatorname{OS}\left(\mathrm{TS}_{3}, \lambda\right)>$ $\mathrm{OS}\left(\mathrm{TS}_{1}, \lambda\right)$, i.e., $\mathrm{TS}_{3}>\mathrm{TS}_{1}$

(3) If $\lambda=0.98$, then $\operatorname{OS}\left(\mathrm{TS}_{1}, \lambda\right)=\operatorname{OS}\left(\mathrm{TS}_{3}, \lambda\right)$ holds. In this case, the following multiplicative accuracy values should be considered:

$$
\begin{aligned}
& A_{0.98}\left(\tilde{d}_{11}\right)=0.289, \\
& A_{0.98}\left(\tilde{d}_{12}\right)=0.709, \\
& A_{0.98}\left(\tilde{d}_{13}\right)=1.229, \\
& A_{0.98}\left(\widetilde{d}_{14}\right)=0.639, \\
& A_{0.98}\left(\widetilde{d}_{31}\right)=0.639, \\
& A_{0.98}\left(\widetilde{d}_{32}\right)=0.805, \\
& A_{0.98}\left(\tilde{d}_{33}\right)=0.709, \\
& A_{0.98}\left(\tilde{d}_{34}\right)=0.289 .
\end{aligned}
$$

Step 5. Accordingly, the overall multiplicative accuracy values of alternatives $\mathrm{TS}_{1}$ and $\mathrm{TS}_{3}$ are calculated as 


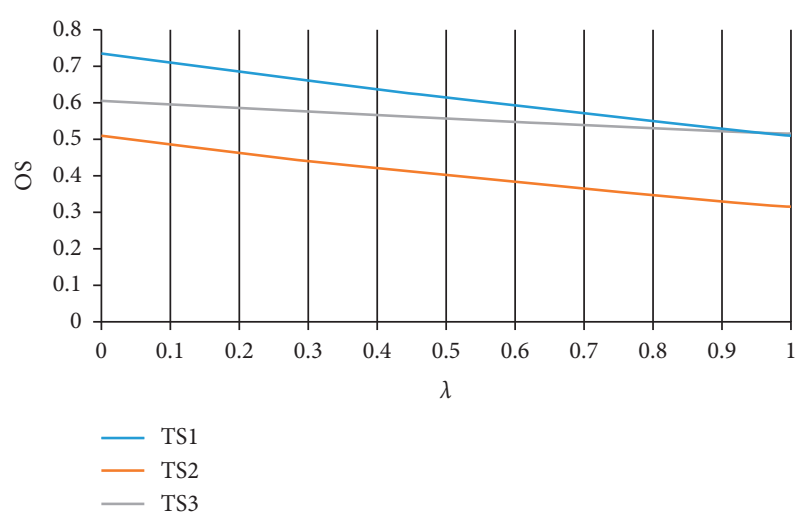

Figure 3: The overall multiplicative score values of three alternatives.

$$
\begin{aligned}
& \mathrm{OA}\left(\mathrm{TS}_{1}, 0.98\right)=\prod_{j=1}^{4} A_{0.98}\left(\widetilde{d}_{1 j}\right)^{\omega_{j}}=0.749 \\
& \mathrm{OA}\left(\mathrm{TS}_{3}, 0.98\right)=\prod_{j=1}^{4} A_{0.98}\left(\widetilde{d}_{3 j}\right)^{\omega_{j}}=0.617
\end{aligned}
$$

It is apparent that $\mathrm{OA}\left(\mathrm{TS}_{1}, 0.98\right)>\mathrm{OA}\left(\mathrm{TS}_{3}, 0.98\right)$. Based on Definition 7 , we have $\mathrm{TS}_{1}>\mathrm{TS}_{3}$. In sum, the ranking order of alternatives $\mathrm{TS}_{1}, \mathrm{TS}_{2}$, and $\mathrm{TS}_{3}$ is shown as follows: (1) if $\lambda \in[0,0.98]$, then $\mathrm{TS}_{1}>\mathrm{TS}_{3}>\mathrm{TS}_{2}$; (2) if $\lambda \in(0.98,1]$, then $\mathrm{TS}_{3}>\mathrm{TS}_{1}>\mathrm{TS}_{2}$.

5.2. Comparison with Xia's Method. Suppose we need to rank three intuitionistic multiplicative numbers $\widetilde{\alpha}_{1}=(3,5)$, $\widetilde{\alpha}_{2}=(1,3)$, and $\widetilde{\alpha}_{3}=(2,6)$ in descending order. Using (31), the multiplicative score values of $\widetilde{\alpha}_{1}, \widetilde{\alpha}_{2}$, and $\widetilde{\alpha}_{3}$ are obtained as

$$
\begin{aligned}
& S_{\lambda}\left(\widetilde{\alpha}_{1}\right)=1.216 \cdot 0.405^{\lambda}, \\
& S_{\lambda}\left(\widetilde{\alpha}_{2}\right)=0.693 \cdot 0.693^{\lambda}, \\
& S_{\lambda}\left(\widetilde{\alpha}_{3}\right)=0.874 \cdot 0.437^{\lambda} .
\end{aligned}
$$

For all $\lambda \in[0,1]$, we have $S_{\lambda}\left(\widetilde{\alpha}_{1}\right)>S_{\lambda}\left(\widetilde{\alpha}_{2}\right)$ and $S_{\lambda}\left(\widetilde{\alpha}_{1}\right)>S_{\lambda}\left(\widetilde{\alpha}_{3}\right)$. From Definition 7, it follows that $\widetilde{\alpha}_{1}>_{\lambda} \widetilde{\alpha}_{2}$ and $\tilde{\alpha}_{1}>_{\lambda} \widetilde{\alpha}_{3}$. Furthermore, if $\lambda \in[0,0.5)$, then $S_{\lambda}\left(\widetilde{\alpha}_{3}\right)>S_{\lambda}\left(\widetilde{\alpha}_{2}\right)$. Accordingly, we have $\tilde{\alpha}_{3}>_{\lambda} \tilde{\alpha}_{2}$. If $\lambda \in(0.5,1]$, then $S_{\lambda}\left(\widetilde{\alpha}_{2}\right)>S_{\lambda}\left(\widetilde{\alpha}_{3}\right)$. Therefore, we have $\widetilde{\alpha}_{2}>_{\lambda} \widetilde{\alpha}_{3}$. Considering $S_{0.5}\left(\widetilde{\alpha}_{2}\right)=S_{0.5}\left(\widetilde{\alpha}_{3}\right)$, we calculate the multiplicative accuracy values $A_{0.5}\left(\widetilde{\alpha}_{2}\right)=1.201$ and $A_{0.5}\left(\widetilde{\alpha}_{3}\right)=1.513$. Based on Definition 7, we have $\widetilde{\alpha}_{3}>_{0.5} \widetilde{\alpha}_{2}$.

In sum, the ranking order of $\widetilde{\alpha}_{1}, \widetilde{\alpha}_{2}$, and $\widetilde{\alpha}_{3}$ can be concluded in two cases: (1) if $\lambda \in[0,0.5]$, then $\widetilde{\alpha}_{1}>\widetilde{\alpha}_{3}>\widetilde{\alpha}_{2} ;$ (2) if $\lambda \in(0.5,1]$, then $\widetilde{\alpha}_{1}>\widetilde{\alpha}_{2}>\widetilde{\alpha}_{3}$.

For comparing with the proposed ranking method, Xia's method [16] is employed to rank intuitionistic multiplicative numbers $\widetilde{\alpha}_{1}, \widetilde{\alpha}_{2}$, and $\widetilde{\alpha}_{3}$. By (2), the multiplicative score values of $\tilde{\alpha}_{1}, \widetilde{\alpha}_{2}$, and $\widetilde{\alpha}_{3}$ are calculated as $S_{\mathrm{Xia}}\left(\widetilde{\alpha}_{1}\right)=(3 / 5)$, $S_{\mathrm{Xia}}\left(\widetilde{\alpha}_{2}\right)=(1 / 3)$, and $S_{\mathrm{Xia}}\left(\widetilde{\alpha}_{3}\right)=(1 / 3)$. Moreover, from (3), we have $A_{\mathrm{Xia}}\left(\widetilde{\alpha}_{2}\right)=3$ and $A_{\mathrm{Xia}}\left(\widetilde{\alpha}_{3}\right)=12$. According to Definition 3, the ranking order is determined as $\widetilde{\alpha}_{1}>\widetilde{\alpha}_{3}>\widetilde{\alpha}_{2}$. The comparative results of two ranking methods are illustrated in Table 1.
TABLE 1: Comparative results of two ranking methods.

\begin{tabular}{lcc}
\hline Ranking method & Parameter & Ranking order \\
\hline Xia's method & Null & $\widetilde{\alpha}_{1}>\widetilde{\alpha}_{3}>\widetilde{\alpha}_{2}$ \\
Proposed method & $\lambda \in[0,0.5]$ & $\widetilde{\alpha}_{1}>\widetilde{\alpha}_{3}>\widetilde{\alpha}_{2}$ \\
Proposed method & $\lambda \in(0.5,1]$ & $\widetilde{\alpha}_{1}>\widetilde{\alpha}_{2}>\widetilde{\alpha}_{3}$ \\
\hline
\end{tabular}

From Table 1, it is obvious that the ranking result of our method is the same as Xia's method when $\lambda \in[0,0.5]$. On the other hand, the ranking results of the two methods are different when $\lambda \in(0.5,1]$. An effective ranking method should consider the risk preference of decision makers. It should be pointed out that Xia's ranking method is a special case of the proposed ranking method. Xia's method only considers the single point value and ignores the infinite number of potential possible values in feasible region $R_{\alpha}$. As a result, the ranking result of Xia's method may be distorted and invalid in some cases. The proposed ranking method in this study considers the risk preference of decision makers in ranking results and traverses all potential possible values. Therefore, the ranking results of our method are more reasonable and effective.

\section{Conclusion and Further Study}

In multiattribute decision-making problems under intuitionistic multiplicative environment, the ranking of fuzzy numbers is directly related to the priority of alternatives. Due to the limitation of resources, knowledge level, and behavior habits, decision makers tend to have different preferences for risk. In this study, a novel and effective ranking method of intuitionistic multiplicative numbers is proposed by defining the multiplicative score function and accuracy function, which integrates the risk preference information of the decision maker. We verify the necessary properties of multiplicative score function and accuracy function and then establish the total order relation of intuitionistic multiplicative numbers. The main advantages and innovations of the proposed ranking method are stated as follows:

(1) The proposed ranking method takes all the potential possible values in feasible region into account. Therefore, the ranking results obtained by our method are more reliable and reasonable. Xia's method only considers the single point value and ignores infinite number of possible values. The ranking result of Xia's method may be distorted and invalid in some cases.

(2) The ranking method proposed in this study integrates the risk preference of decision makers. The ranking results are more flexible and effective than Xia's method. We prove that Xia's ranking method is a special case of the proposed ranking method.

(3) We establish the total order relation of intuitionistic multiplicative numbers with risk preference information of decision makers. The necessary axiomatic properties (asymmetry, irreflexivity, and transitivity) of total order relation are verified. 
In addition, the proposed ranking method is applied to select the optimal logistics transfer station with intuitionistic multiplicative information. A comparison example is developed to highlight the advantage and effectiveness of the proposed ranking method. In the future, we consider extending the ranking method to nonequilibrium multiplicative environment. We will try to apply the series ranking method to deal with practical problems such as pollution control, system assessment, and energy management.

\section{Data Availability}

The data used to support the findings of this study are included within the article.

\section{Conflicts of Interest}

The authors declare that they have no conflicts of interest.

\section{Acknowledgments}

This work was supported by the National Natural Science Foundation of China (No. 71601049), the Humanities and Social Sciences Fund of the Ministry of Education (No. 16YJC630064), the Natural Science Foundation of Fujian Province (No. 2020J01576), the Foundation of Beijing Intelligent Logistics System Collaborative Innovation Center (No. BILSCIC-2019KF-16), China Postdoctoral Science Foundation (No. 2019M660242), the Construction Fund for Digital Fujian Big Data for Agriculture and Forestry (No. KJG18019A), and the University Training Program in Scientific Research for Outstanding Young Talents of Fujian Province: COST sharing strategy of incomplete cooperative games and its application in water pollution control (No. K80SCC53A).

\section{References}

[1] K. T. Atanassov, "Intuitionistic fuzzy sets," Fuzzy Sets and Systems, vol. 20, no. 1, pp. 87-96, 1986.

[2] P. Hajek, W. Froelich, and O. Prochazka, "Intuitionistic fuzzy grey cognitive maps for forecasting interval-valued time series," Neurocomputing, vol. 400, pp. 173-185, 2020.

[3] M. Lin, Z. Chen, H. Liao, and Z. Xu, "ELECTRE II method to deal with probabilistic linguistic term sets and its application to edge computing," Nonlinear Dynamics, vol. 96, no. 3, pp. 2125-2143, 2019.

[4] M. W. Lin, C. Huang, Z. S. Xu, and R. Q. Chen, "Evaluating IoT platforms using integrated probabilistic linguistic MCDM method," IEEE Internet of Things Journal, vol. 7, no. 11, pp. 11195-11208, 2020.

[5] Y. K. Dubey, M. M. Mushrif, and K. Mitra, "Segmentation of brain MR images using rough set based intuitionistic fuzzy clustering," Biocybernetics and Biomedical Engineering, vol. 36, no. 2, pp. 413-426, 2016.

[6] H. Verma, R. K. Agrawal, and A. Sharan, "An improved intuitionistic fuzzy $c$-means clustering algorithm incorporating local information for brain image segmentation," Applied Soft Computing, vol. 46, pp. 543-557, 2016.

[7] X. X. Lei, H. L. Ouyang, and L. J. Xu, "Kernel distance-based intuitionistic fuzzy $c$-means clustering algorithm and its application," Pattern Recognition and Image Analysis, vol. 29, pp. 592-597, 2019.

[8] M. Lin, Z. Xu, Y. Zhai, and Z. Yao, "Multi-attribute group decision-making under probabilistic uncertain linguistic environment," Journal of the Operational Research Society, vol. 69, no. 2, pp. 157-170, 2018.

[9] L. Wang and N. Li, "Pythagorean fuzzy interaction power Bonferroni mean aggregation operators in multiple attribute decision making," International Journal of Intelligent Systems, vol. 35, no. 1, pp. 150-183, 2020.

[10] M. W. Lin, C. Huang, Z. S. Xu, and R. Q. Chen, "Pythagorean fuzzy MULTIMOORA method based on distance measure and score function: its application in multicriteria decisionmaking process," Knowledge and Information Systems, vol. 62, pp. 4373-4406, 2020.

[11] J. Lin, F. Meng, R. Chen, and Q. Zhang, "Preference attitudebased method for ranking intuitionistic fuzzy numbers and its application in renewable energy selection," Complexity, vol. 2018, Article ID 6251384, 14 pages, 2018.

[12] M. Lin, C. Huang, and Z. Xu, "MULTIMOORA based MCDM model for site selection of car sharing station under picture fuzzy environment," Sustainable Cities and Society, vol. 53, Article ID 101873, 2020.

[13] D. R. Babak and Y. Abdullah, "Intuitionistic Fuzzy TOPSIS method for green supplier selection problem," Soft Computing, vol. 3, pp. 2215-2228, 2020.

[14] Z.-P. Tian, H.-Y. Zhang, J.-Q. Wang, and T.-L. Wang, "Green supplier selection using improved TOPSIS and best-worst method under intuitionistic fuzzy environment," Informatica, vol. 29, no. 4, pp. 773-800, 2018.

[15] L. Xiao et al., "Green supplier selection in steel industry with intuitionistic fuzzy taxonomy method," Journal of Intelligent \& Fuzzy Systems, vol. 1, pp. 1-12, 2020.

[16] M. M. Xia, Z. S. Xu, and H. C. Liao, "Preference relations based on intuitionistic multiplicative information," IEEE Transactions on Fuzzy Systems, vol. 21, pp. 113-133, 2013.

[17] M. Xia and Z. Xu, "Group decision making based on intuitionistic multiplicative aggregation operators," Applied Mathematical Modelling, vol. 37, no. 7, pp. 5120-5133, 2013.

[18] Y. Jiang, Z. Xu, and X. Yu, "Compatibility measures and consensus models for group decision making with intuitionistic multiplicative preference relations," Applied Soft Computing, vol. 13, no. 4, pp. 2075-2086, 2013.

[19] Y. Jiang, Z. Xu, and X. Yu, "Group decision making based on incomplete intuitionistic multiplicative preference relations," Information Sciences, vol. 295, pp. 33-52, 2015.

[20] Z. S. Xu, "Priority weight intervals derived from intuitionistic multiplicative preference relations," IEEE Transactions on Fuzzy Systems, vol. 21, no. 4, pp. 642-654, 2013.

[21] Z. S. Xu and M. M. Xia, "Group decision making based on intuitionistic multiplicative aggregation operators," Applied Mathematical Modelling, vol. 37, no. 7, pp. 5120-5133, 2013.

[22] Y. Jiang and Z. Xu, "Aggregating information and ranking alternatives in decision making with intuitionistic multiplicative preference relations," Applied Soft Computing, vol. 22, pp. 162-177, 2014.

[23] D. Yu and L. Fang, "Intuitionistic multiplicative aggregation operators with their application in group decision making," Journal of Intelligent \& Fuzzy Systems, vol. 27, no. 1, pp. 131-142, 2014.

[24] S. Yu and Z. Xu, "Aggregation and decision making using intuitionistic multiplicative triangular fuzzy information," Journal of Systems Science and Systems Engineering, vol. 23, no. 1, pp. 20-38, 2014. 
[25] Y. Jiang, Z. Xu, and J. Xu, "Interval-valued intuitionistic multiplicative sets," International Journal of Uncertainty, Fuzziness and Knowledge-Based Systems, vol. 22, no. 03, pp. 385-406, 2014.

[26] P. Ren, Z. Xu, and H. Liao, "Intuitionistic multiplicative analytic hierarchy process in group decision making," Computers \& Industrial Engineering, vol. 101, pp. 513-524, 2016.

[27] W. Qian and L. Niu, "Intuitionistic multiplicative preference relation and its application in group decision making," Journal of Intelligent \& Fuzzy Systems, vol. 30, no. 5, pp. 2859-2870, 2016.

[28] Y. Jiang, Z. S. Xu, and Y. H. Shu, "Distance measures between intuitionistic multiplicative sets and their application in selection of satellite earth stations," System Engineering Theory and Practice, vol. 36, no. 12, pp. 3210-3219, 2016.

[29] H. Garg, "Distance and similarity measures for intuitionistic multiplicative preference relation and its applications," International Journal for Uncertainty Quantification, vol. 7, no. 2, pp. 117-133, 2017.

[30] Z. Zhang and W. Pedrycz, "Models of mathematical programming for intuitionistic multiplicative preference relations," IEEE Transactions on Fuzzy Systems, vol. 25, no. 4, pp. 945-957, 2017.

[31] Z. Zhang and C. Guo, "Deriving priority weights from intuitionistic multiplicative preference relations under group decision-making settings," Journal of the Operational Research Society, vol. 68, no. 12, pp. 1582-1599, 2017.

[32] Z. M. Ma and Z. S. Xu, "Hyperbolic scales involving appetitesbased intuitionistic multiplicative preference relations for group decision making," Information Sciences, vol. 451-452, pp. 310-325, 2018.

[33] H. Liao, C. Zhang, and L. Luo, "A multiple attribute group decision making method based on two novel intuitionistic multiplicative distance measures," Information Sciences, vol. 467, pp. 766-783, 2018.

[34] F. Jin, Z. Ni, L. Pei, H. Chen, and Y. Li, "Goal programming approach to derive intuitionistic multiplicative weights based on intuitionistic multiplicative preference relations," International Journal of Machine Learning and Cybernetics, vol. 9, no. 4, pp. 641-650, 2018.

[35] Z. Zhang and W. Pedrycz, "Intuitionistic multiplicative group analytic hierarchy process and its use in multicriteria group decision-making," IEEE Transactions on Cybernetics, vol. 48, no. 7, pp. 1950-1962, 2018.

[36] S. Mamata, "Acceptably consistent incomplete interval-valued intuitionistic multiplicative preference relations," Soft Computing, vol. 22, no. 22, pp. 7463-7477, 2018.

[37] Z. Zhang, S.-M. Chen, and C. Wang, "Group decision making with incomplete intuitionistic multiplicative preference relations," Information Sciences, vol. 516, pp. 560-571, 2020.

[38] Y. Jiang, Z. Xu, and M. Gao, "Methods for ranking intuitionistic multiplicative numbers by distance measures in decision making," Computers \& Industrial Engineering, vol. 88, pp. 100-109, 2015.

[39] T.-Y. Chen, "A comparative analysis of score functions for multiple criteria decision making in intuitionistic fuzzy settings," Information Sciences, vol. 181, no. 17, pp. 3652-3676, 2011. 